\title{
Remerciements aux relecteurs 2021
}

\section{Carmen Renaudeau}

La revue Cahiers Agricultures remercie tous les relecteurs qui ont bien voulu l'aider à prendre une décision sur la publication des textes et ont fourni aux auteurs de précieux conseils.

\author{
Hervé Aholoukpè \\ Mikaël Akimowicz \\ Céline Allaverdian \\ Nadine Andrieu \\ Aminou Arouna \\ Juan Barrera \\ Imed Ben Aissa \\ David Berre \\ Cécile Bessou \\ Jérôme Bindelle \\ Stéphane Blancard \\ Vincent Blanfort \\ Boualem Brahim Bouammar \\ Emmanuelle Bouquet \\ Aurélie Carimentrand \\ François Casabianca \\ Antonio Castellanos-Navarrete \\ Jean-Daniel Cesaro \\ Hubert Cochet \\ Nicole Darmon \\ Hubert de Bon \\ Chris de Bont \\ Marcel de Raissac \\ Marie-Sophie Dedieu \\ Julien Demenois \\ Soazig Di Bianco \\ Djiby Dia \\ Jean-Christophe Diepart \\ François Doligez \\ Olivier Ducourtieux \\ Patrick Dugué \\ Bertrand Dumont \\ Tristan Durand-Gasselin \\ Sandrine Dury \\ Fatima El Hadad Gauthier \\ Mohamed Elloumi \\ Mathilde Fautras \\ Nicolas Faysse \\ Laurene Feintrenie \\ Anne Floquet \\ Stéphane Fournier \\ Amandine Gautier \\ Frédéric Gay \\ Kouame Casimir Gboko
}

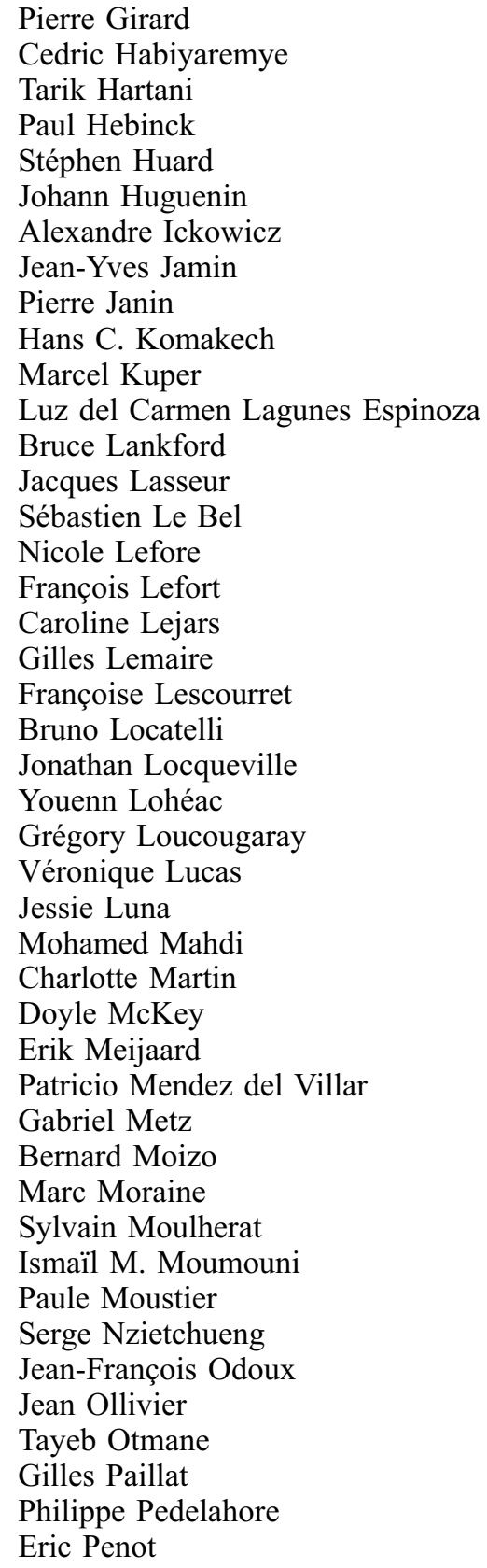

Pierre Girard

Cedric Habiyaremye

Tarik Hartani

Paul Hebinck

Stéphen Huard

Johann Huguenin

Alexandre Ickowicz

Jean-Yves Jamin

Pierre Janin

Hans C. Komakech

Marcel Kuper

Luz del Carmen Lagunes Espinoza

Bruce Lankford

Jacques Lasseur

Sébastien Le Bel

Nicole Lefore

François Lefort

Caroline Lejars

Gilles Lemaire

Françoise Lescourret

Bruno Locatelli

Jonathan Locqueville

Youenn Lohéac

Grégory Loucougaray

Véronique Lucas

Jessie Luna

Mohamed Mahdi

Charlotte Martin

Doyle McKey

Erik Meijaard

Patricio Mendez del Villar

Gabriel Metz

Bernard Moizo

Marc Moraine

Sylvain Moulherat

Ismaïl M. Moumouni

Paule Moustier

Serge Nzietchueng

Jean-François Odoux

Jean Ollivier

Tayeb Otmane

Gilles Paillat

Philippe Pedelahore

Eric Penot 
Cah. Agric. 2022, 31, 5

Samuel Yves Perichon

Anne Perinelle

Sylvain Perret

Lorène Prost

Angèle Proust

Sylvain Rafflegeau

Soanandrasana Rahelinirina

Christine Rawski

Emmanuel Raynaud

Stephane François

Dominique Rennesson

Jeanne Riaux

Julie Rivière

Marie-Hélène Robin-Ext

Max Rousseau

Samira Rousselière

Simon R. Ruegg

Eric Sabourin
Chloé Salembier

Mohamed Taher Sraïri

Abdoulaye Tapsoba

Pascal Thiébeau

Emmanuel Torquebiau

Jean-François Tourrand

Jean-Marc Touzard

Isabelle Tritsch

Elodie Valette

Bertrand Valiorgue

Eric Vall

Arielle Vidal

Robin Villemaine-Bessière

Florence Volaire

Philip Woodhouse

Sibiri Jean Zoundi

Margreet Zwarteveen

Citation de l'article : Renaudeau C. 2022. Remerciements aux relecteurs 2021. Cah. Agric. 31: 5. 